\title{
Tumor Pathophysiology studied with Multiphoton Laser-Scanning Microscopy
}

\author{
Edward Brown \\ Edwin L. Steele Laboratory for Tumor Biology \\ Department of Radiation Oncology, Cox 7 \\ Massachusetts General Hospital \\ Harvard University Medical School \\ Boston, MA 02114
}

The Multiphoton-Laser Scanning Microscope (MPLSM) has had a major impact on the field of tumor pathophysiology. The superior depth penetration of the (MPLSM) allows in vivo exploration of the tumor and its microenvironment, while the minimal photodamage of the technique allows long-term dynamic studies. The intrinsic contrast mechanism of second harmonic generation can be used in the MPLSM to image collagen in the tumor extracellular matrix. We present recent studies of cell behavior, tumorinduced gene promoter activity, and the tumor extracellular matrix. We focus on dynamic activity of host stromal cells in the tumor, dynamic alteration of the tumor vasculature by therapeutic agents, and dynamic modification of the tumor extracellular matrix by hormonal therapy. 


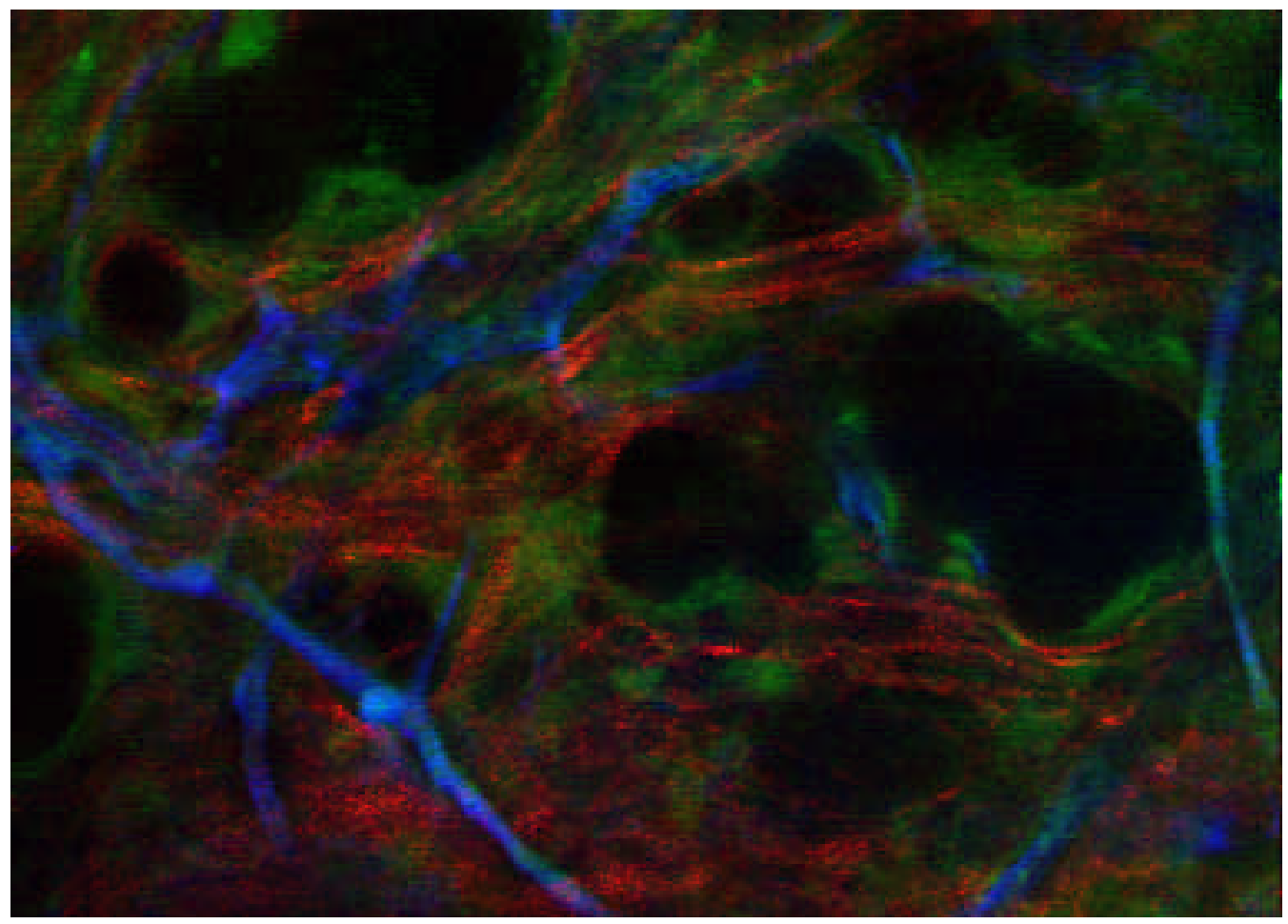

Two Photon Microscopy of Tumors in Vivo. This is a single optical section of an MCaIV murine mammary adenocarcinoma growing in the dorsal skinfold chamber of a VEGFGFP transgenic mouse. Green represents GFP expressing host stromal cells that have penetrated the tumor, blue represents blood vessels labeled with i.v. injection of TMRdextran, and red represents fibrillar collagen imaged with second harmonic generation. The black circles represent clusters of tumor cells that have not been penetrated by GFPexpressing host stromal cells, blood vessels, or significant collagen fibers. Image is 500 microns across. 